\title{
VISCOELASTIC FRICTIONLESS CONTACT PROBLEMS WITH ADHESION
}

\author{
MOHAMED SELMANI AND MIRCEA SOFONEA
}

Received 16 December 2005; Revised 8 March 2006; Accepted 9 March 2006

We consider two quasistatic frictionless contact problems for viscoelastic bodies with long memory. In the first problem the contact is modelled with Signorini's conditions and in the second one is modelled with normal compliance. In both problems the adhesion of the contact surfaces is taken into account and is modelled with a surface variable, the bonding field. We provide variational formulations for the mechanical problems and prove the existence of a unique weak solution to each model. The proofs are based on arguments of time-dependent variational inequalities, differential equations, and a fixed point theorem. Moreover, we prove that the solution of the Signorini contact problem can be obtained as the limit of the solutions of the contact problem with normal compliance as the stiffness coefficient of the foundation converges to infinity.

Copyright (c) 2006 M. Selmani and M. Sofonea. This is an open access article distributed under the Creative Commons Attribution License, which permits unrestricted use, distribution, and reproduction in any medium, provided the original work is properly cited.

\section{Introduction}

The adhesive contact between deformable bodies, when a glue is added to prevent relative motion of the surfaces, has received recently increased attention in the mathematical literature. Basic modelling can be found in [7-9, 12, 17]. Analysis of models for adhesive contact can be found in $[1-6,10]$ and in the recent monographs $[15,16]$. An application of the theory of adhesive contact in the medical field of prosthetic limbs was considered in $[13,14]$; there, the importance of the bonding between the bone-implant and the tissue was outlined, since debonding may lead to decrease in the persons ability to use the artificial limb or joint.

The novelty in all the above papers is the introduction of a surface internal variable, the bonding field, denoted in this paper by $\beta$; it describes the pointwise fractional density of active bonds on the contact surface, and sometimes referred to as the intensity of adhesion. Following $[7,8]$, the bonding field satisfies the restrictions $0 \leq \beta \leq 1$; when $\beta=1$ at a point of the contact surface, the adhesion is complete and all the bonds are active; when

Hindawi Publishing Corporation Journal of Inequalities and Applications Volume 2006, Article ID 36130, Pages 1-22 DOI 10.1155/JIA/2006/36130 
$\beta=0$ all the bonds are inactive, severed, and there is no adhesion; when $0<\beta<1$ the adhesion is partial and only a fraction $\beta$ of the bonds is active. We refer the reader to the extensive bibliography on the subject in $[9,12,13,15,16]$.

The aim of this paper is to continue the study of adhesive problems begun in $[3,4,6]$. There, models for dynamic or quasistatic process of frictionless adhesive contact between a deformable body and a foundation have been analyzed and simulated; the contact was described with normal compliance or was assumed to be bilateral, and the behavior of the material was modelled with a nonlinear Kelvin-Voigt viscoelastic constitutive law; the models included the bonding field as an additional dependent variable, defined and evolving on the contact surface; the existence of a unique weak solution to the models has been obtained by using arguments of evolutionary equations in Banach spaces and a fixed point theorem.

In this paper we study two quasistatic problems of frictionless adhesive contact. The novelty with respect to the papers referred to in the previous paragraph consists in the fact that here we model the material's behavior with a viscoelastic constitutive law with long memory and the contact with Signorini's conditions or with normal compliance. We derive a variational formulation of the problems and prove the existence of a unique weak solution to each one. To this end, we use similar arguments as in $[3,4,6]$ but with a different choice of functionals and operators, since the constitutive law and the contact boundary conditions, here and in the above-mentioned papers, are different. Moreover, we study the behavior of the solutions of the problem with normal compliance as the stiffness coefficient of the foundation tends to infinity.

The paper is structured as follows. In Section 2, we present some notation and preliminary material. In Section 3, we state the mechanical models of viscoelastic frictionless contact with adhesion, list the assumptions on the data, and derive their variational formulation. In Sections 4 and 5, we present our main existence and uniqueness results, Theorems 4.1 and 5.1, which state the unique weak solvability of the adhesive frictionless contact problem with Signorini and normal compliance conditions, respectively. Finally, in Section 6 we prove a convergence result, Theorem 6.1; it states that the solution of the adhesive contact problem with normal compliance converges to the solution of the adhesive Signorini contact problem as the stiffness coefficient of the foundation converges to infinity.

\section{Notations and preliminaries}

Everywhere in this paper we denote by $\mathbb{S}^{d}$ the space of second-order symmetric tensors on $\mathbb{R}^{d}(d=1,2,3)$, while "." and $\|\cdot\|$ represent the inner product and the Euclidean norm on $\mathbb{R}^{d}$ and $\mathbb{S}^{d}$, respectively. Thus, for every $\mathbf{u}, \mathbf{v} \in \mathbb{R}^{d}$ and $\boldsymbol{\sigma}, \boldsymbol{\tau} \in \mathbb{S}^{d}$ we have

$$
\mathbf{u} \cdot \mathbf{v}=u_{i} v_{i}, \quad\|\mathbf{v}\|=(\mathbf{v} \cdot \mathbf{v})^{1 / 2}, \quad \boldsymbol{\sigma} \cdot \boldsymbol{\tau}=\sigma_{i j} \tau_{i j}, \quad\|\boldsymbol{\tau}\|=(\boldsymbol{\tau} \cdot \boldsymbol{\tau})^{1 / 2} .
$$

Here and below, the indices $i, j, k, l$ run between 1 and $d$ and the summation convention over repeated indices is adopted.

Let $\Omega \subset \mathbb{R}^{d}$ be a bounded domain with a Lipschitz continuous boundary $\Gamma$. In what follows we use the standard notation for the $L^{p}$ and Sobolev spaces associated to $\Omega$ and $\Gamma$, 
and the index that follows a comma indicates a derivative with respect to the corresponding component of the spatial variable $\mathbf{x} \in \Omega$. We also use the spaces

$$
\begin{gathered}
H_{1}=H^{1}(\Omega)^{d}=\left\{\mathbf{u}=\left(u_{i}\right): u_{i} \in H^{1}(\Omega)\right\}, \\
Q=\left\{\boldsymbol{\sigma}=\left(\sigma_{i j}\right): \sigma_{i j}=\sigma_{j i} \in L^{2}(\Omega)\right\}, \\
Q_{1}=\left\{\boldsymbol{\sigma} \in Q: \sigma_{i j, j} \in L^{2}(\Omega)\right\} .
\end{gathered}
$$

These are real Hilbert spaces endowed with the inner products given by

$$
\begin{gathered}
(\mathbf{u}, \mathbf{v})_{H_{1}}=\int_{\Omega} \mathbf{u} \cdot \mathbf{v} d x+\int_{\Omega} \boldsymbol{\varepsilon}(\mathbf{u}) \cdot \boldsymbol{\varepsilon}(\mathbf{v}) d x \\
(\boldsymbol{\sigma}, \boldsymbol{\tau})_{Q}=\int_{\Omega} \boldsymbol{\sigma} \cdot \boldsymbol{\tau} d x \\
(\boldsymbol{\sigma}, \boldsymbol{\tau})_{Q_{1}}=\int_{\Omega} \boldsymbol{\sigma} \cdot \boldsymbol{\tau} d x+\int_{\Omega} \operatorname{Div} \boldsymbol{\sigma} \cdot \operatorname{Div} \boldsymbol{\tau} d x,
\end{gathered}
$$

respectively, where $\boldsymbol{\varepsilon}: H^{1}(\Omega)^{d} \rightarrow Q$ and Div : $Q_{1} \rightarrow L^{2}(\Omega)^{d}$ are the deformation and the divergence operators defined by

$$
\boldsymbol{\varepsilon}(\mathbf{u})=\left(\varepsilon_{i j}(\mathbf{u})\right), \quad \varepsilon_{i j}(\mathbf{u})=\frac{1}{2}\left(u_{i, j}+u_{j, i}\right), \quad \operatorname{Div} \boldsymbol{\sigma}=\left(\sigma_{i j, j}\right) .
$$

The associated norms on the spaces $H_{1}, Q$, and $Q_{1}$ are denoted by $\|\cdot\|_{H_{1}},\|\cdot\|_{Q}$, and $\|\cdot\|_{Q_{1}}$, respectively.

Since the boundary $\Gamma$ is Lipschitz continuous, the unit outward normal vector $\boldsymbol{v}$ on the boundary is defined almost everywhere. For every vector field $\mathbf{v} \in H_{1}$ we use the notation $\mathbf{v}$ for the trace of $\mathbf{v}$ on $\Gamma$ and we denote by $v_{v}$ and $\mathbf{v}_{\tau}$ the normal and the tangential components of $\mathbf{v}$ on the boundary, given by

$$
v_{\nu}=\mathbf{v} \cdot \boldsymbol{v}, \quad \mathbf{v}_{\tau}=\mathbf{v}-v_{\nu} \boldsymbol{\nu} .
$$

For a regular ( say $C^{1}$ ) stress field $\sigma$, the application of its trace on the boundary to $\boldsymbol{v}$ is the Cauchy stress vector $\sigma v$. We define, similarly, the normal and tangential components of the stress on the boundary by the formulas

$$
\sigma_{\nu}=(\sigma \boldsymbol{\nu}) \cdot \boldsymbol{\nu}, \quad \sigma_{\tau}=\sigma \boldsymbol{\nu}-\sigma_{\nu} \boldsymbol{\nu}
$$

and we recall that the following Green's formula holds:

$$
\int_{\Omega} \boldsymbol{\sigma} \cdot \boldsymbol{\varepsilon}(\mathbf{v}) d x+\int_{\Omega} \operatorname{Div} \boldsymbol{\sigma} \cdot \mathbf{v} d x=\int_{\Gamma} \boldsymbol{\sigma} \boldsymbol{\nu} \cdot \mathbf{v} d a \quad \forall \mathbf{v} \in H_{1}
$$

For every real Banach space $\left(X,\|\cdot\|_{X}\right)$ and $T>0$ we use the classical notation for the spaces $L^{p}(0, T ; X)$ and $W^{k, p}(0, T ; X)$ where $1 \leq p \leq+\infty, k=1,2, \ldots$, and we denote by 
4 Viscoelastic frictionless contact problems with adhesion

$C([0, T] ; X)$ the space of continuous functions on $[0, T]$ with values on $X$, with the norm

$$
\|x\|_{C([0, T] ; X)}=\max _{t \in[0, T]}\|x(t)\|_{X} .
$$

Moreover, we use the dot above to indicate the derivative with respect to the time variable and, for a real number $r$, we use $r_{+}$to represent its positive part, that is, $r_{+}=\max \{0, r\}$.

\section{Problems statement}

We consider a viscoelastic body which occupies the domain $\Omega \subset \mathbb{R}^{d}$, and assume that its boundary $\Gamma$ is divided into three disjoint measurable parts $\Gamma_{1}, \Gamma_{2}$, and $\Gamma_{3}$ such that meas $\Gamma_{1}>0$. Let $T>0$ and let $[0, T]$ denote the time interval of interest. The body is clamped on $\Gamma_{1} \times(0, T)$ and, therefore, the displacement field vanishes there. A volume force of density $\mathbf{f}_{0}$ acts in $\Omega \times(0, T)$ and surface tractions of density $\mathbf{f}_{2}$ act on $\Gamma_{2} \times(0, T)$. The body is in an adhesive frictionless contact with an obstacle, the so-called foundation, over the potential contact surface $\Gamma_{3}$. Moreover, the process is quasistatic, that is, the inertial terms are neglected in the equation of motion. We use a linearly viscoelastic constitutive law with long memory to model the material's behavior and an ordinary differential equation to describe the evolution of the bonding field.

For the first problem, we consider here the contact is modelled with Signorini's conditions with adhesion. Thus, the classical model for the process is the following.

Problem 3.1. Find a displacement field $\mathbf{u}: \Omega \times[0, T] \rightarrow \mathbb{R}^{d}$, a stress field $\boldsymbol{\sigma}: \Omega \times[0, T] \rightarrow$ $\mathbb{S}^{d}$, and a bonding field $\beta: \Gamma_{3} \times[0, T] \rightarrow[0,1]$ such that, for all $t \in[0, T]$,

$$
\begin{aligned}
& \boldsymbol{\sigma}(t)=\mathscr{A} \boldsymbol{\varepsilon}(\mathbf{u}(t))+\int_{0}^{t} \mathscr{B}(t-s) \boldsymbol{\varepsilon}(\mathbf{u}(s)) d s \quad \text { in } \Omega, \\
& \operatorname{Div} \boldsymbol{\sigma}(t)+\mathbf{f}_{0}(t)=\mathbf{0} \quad \text { in } \Omega \text {, } \\
& \mathbf{u}(t)=\mathbf{0} \quad \text { on } \Gamma_{1}, \\
& \boldsymbol{\sigma}(t) \boldsymbol{v}=\mathbf{f}_{2}(t) \quad \text { on } \Gamma_{2}, \\
& u_{\nu}(t) \leq 0, \quad \sigma_{\nu}(t)-\gamma_{\nu} R_{\nu}\left(u_{\nu}(t)\right) \beta^{2}(t) \leq 0, \\
& \left(\sigma_{\nu}(t)-\gamma_{\nu} R_{\nu}\left(u_{\nu}(t)\right) \beta^{2}(t)\right) u_{\nu}(t)=0 \quad \text { on } \Gamma_{3} \text {, } \\
& -\boldsymbol{\sigma}_{\tau}(t)=p_{\tau}(\beta(t)) \mathbf{R}_{\tau}\left(\mathbf{u}_{\tau}(t)\right) \quad \text { on } \Gamma_{3}, \\
& \dot{\beta}(t)=-\left(\beta(t)\left(\gamma_{v} R_{\nu}\left(u_{v}(t)\right)^{2}+\gamma_{\tau}\left\|\mathbf{R}_{\tau}\left(\mathbf{u}_{\tau}(t)\right)\right\|^{2}\right)-\epsilon_{a}\right)_{+} \text {on } \Gamma_{3} \text {, } \\
& \beta(0)=\beta_{0} \quad \text { on } \Gamma_{3} \text {. }
\end{aligned}
$$

We now describe the equations and conditions involved in our model above.

First, (3.1) represent the viscoelastic constitutive law with memory, in which $\mathscr{A}$ and $\mathscr{B}$ denote the elasticity and the relaxation fourth-order tensors, respectively. Equation (3.2) is the equilibrium equation while (3.3) and (3.4) are the displacement and traction boundary conditions, respectively. 
Conditions (3.5) represent the Signorini conditions with adhesion where $\gamma_{\nu}$ is a given adhesion coefficient and $R_{v}$ is the truncation operator defined by

$$
R_{v}(s)= \begin{cases}L & \text { if } s<-L \\ -s & \text { if }-L \leq s \leq 0 \\ 0 & \text { if } s>0\end{cases}
$$

Here $L>0$ is the characteristic length of the bond beyond which it does not offer any additional traction. The introduction of the operator $R_{\nu}$, together with the operator $\mathbf{R}_{\tau}$ defined below, is motivated by the mathematical arguments but it is not restrictive for the applied point of view, since no restriction on the size of the parameter $L$ is made in what follows. Thus, by choosing $L$ very large, we can assume that $R_{\nu}\left(u_{\nu}\right)=-u_{\nu}$ and, therefore, from (3.5) we recover the contact conditions

$$
u_{v} \leq 0, \quad \sigma_{\nu}+\gamma_{\nu} u_{v} \beta^{2} \leq 0, \quad\left(\sigma_{\nu}+\gamma_{\nu} u_{v} \beta^{2}\right) u_{v}=0 \quad \text { on } \Gamma_{3} \times(0, T)
$$

These conditions were used in $[5,12]$ to model the unilateral adhesive contact. It follows from (3.5) that there is no penetration between the body and the foundation, since $u_{v} \leq 0$ during the process. Also, note that when the bonding field vanishes, then the contact conditions (3.5) become the classical Signorini contact conditions with zero gap function, that is

$$
u_{\nu} \leq 0, \quad \sigma_{\nu} \leq 0, \quad \sigma_{\nu} u_{v}=0 \quad \text { on } \Gamma_{3} \times(0, T) .
$$

Condition (3.6) represents the adhesive contact condition on the tangential plane in which $p_{\tau}$ is a given function and $\mathbf{R}_{\tau}$ is the truncation operator given by

$$
\mathbf{R}_{\tau}(\mathbf{v})= \begin{cases}\mathbf{v} & \text { if }\|\mathbf{v}\| \leq L, \\ L \frac{\mathbf{v}}{\|\mathbf{v}\|} & \text { if }\|\mathbf{v}\|>L\end{cases}
$$

This condition shows that the shear on the contact surface depends on the bonding field and on the tangential displacement, but as long as it does not exceed the bond length $L$. The frictional tangential traction is assumed to be much smaller than the adhesive one and, therefore, omitted.

Next, (3.7) represents the ordinary differential equation which describes the evolution of the bonding field and it was already used in $[3,5]$, see also $[15,16]$ for more details. Here, besides $\gamma_{\nu}$, two new adhesion coefficients are involved, $\gamma_{\tau}$ and $\epsilon_{a}$, and $R_{\nu}(s)^{2}$ is a short notation for $\left(R_{v}(s)\right)^{2}$, that is, $R_{v}(s)^{2}=\left(R_{\nu}(s)\right)^{2}$. Notice that in this model once debonding occurs, bonding cannot be reestablished since, as it follows from (3.7), $\dot{\beta} \leq 0$.

Finally, (3.8) represents the initial condition in which $\beta_{0}$ is the given initial bonding field.

For the second problem, we study in this paper that the contact is modelled with normal compliance and adhesion, and therefore the classical model for the process is the following. 
6 Viscoelastic frictionless contact problems with adhesion

Problem 3.2. Find a displacement field $\mathbf{u}: \Omega \times[0, T] \rightarrow \mathbb{R}^{d}$, a stress field $\sigma: \Omega \times[0, T] \rightarrow$ $\mathbb{S}^{d}$, and a bonding field $\beta: \Gamma_{3} \times[0, T] \rightarrow[0,1]$ such that, for all $t \in[0, T]$,

$$
\begin{gathered}
\boldsymbol{\sigma}(t)=\mathscr{A} \boldsymbol{\varepsilon}(\mathbf{u}(t))+\int_{0}^{t} \mathscr{B}(t-s) \boldsymbol{\varepsilon}(\mathbf{u}(s)) d s \quad \text { in } \Omega, \\
\operatorname{Div} \boldsymbol{\sigma}(t)+\mathbf{f}_{0}(t)=\mathbf{0} \quad \text { in } \Omega, \\
\mathbf{u}(t)=\mathbf{0} \quad \text { on } \Gamma_{1}, \\
\boldsymbol{\sigma} \boldsymbol{v}(t)=\mathbf{f}_{2}(t) \quad \text { on } \Gamma_{2}, \\
-\sigma_{\nu}(t)=p_{\nu}\left(u_{\nu}(t)\right)-\gamma_{\nu} \beta^{2} R_{\nu}\left(u_{\nu}(t)\right) \quad \text { on } \Gamma_{3}, \\
-\sigma_{\tau}(t)=p_{\tau}(\beta(t)) \mathbf{R}_{\tau}\left(\mathbf{u}_{\tau}(t)\right) \quad \text { on } \Gamma_{3}, \\
\dot{\beta}(t)=-\left(\beta(t)\left(\gamma_{v} R_{v}\left(u_{v}(t)\right)^{2}+\gamma_{\tau}\left\|\mathbf{R}_{\tau}\left(\mathbf{u}_{\tau}(t)\right)\right\|^{2}\right)-\epsilon_{a}\right)_{+} \text {on } \Gamma_{3}, \\
\beta(0)=\beta_{0} \quad \text { on } \Gamma_{3} .
\end{gathered}
$$

Note that the equations and conditions involved in Problem 3.2 have the same meaning as those involved in Problem 3.1. The difference arises from the fact that here we replace Signorini's contact conditions with adhesion, (3.5), with the normal compliance contact condition with adhesion, (3.17), where $p_{\nu}$ is a given positive function which will be described below. In this condition the interpenetrability between the body and the foundation is allowed, that is, $u_{v}$ can be positive on $\Gamma_{3}$. The contribution of the adhesive traction to the normal one is represented by the term $\gamma_{\nu} \beta^{2} R_{\nu}\left(u_{\nu}\right)$; the adhesive traction is tensile and is proportional, with proportionality coefficient $\gamma_{v}$, to the square of the intensity of adhesion and to the normal displacement, but as long as it does not exceed the bond length $L$. The maximal tensile traction is $\gamma_{\nu} L$. The contact condition (3.17) was used in various papers; see, for example, $[3,4,15,16]$ and the references therein.

We turn to the variational formulation of the mechanical Problems 3.1 and 3.2. To this end, for the displacement field we need the closed subspace of $H_{1}$ defined by

$$
V=\left\{\mathbf{v} \in H_{1} \mid \mathbf{v}=\mathbf{0} \text { on } \Gamma_{1}\right\}
$$

Since meas $\Gamma_{1}>0$, Korn's inequality holds; thus, there exists a constant $c_{K}>0$, that depends only on $\Omega$ and $\Gamma_{1}$, such that

$$
\|\boldsymbol{\varepsilon}(\mathbf{v})\|_{\mathscr{H}} \geq c_{K}\|\mathbf{v}\|_{H_{1}} \quad \forall \mathbf{v} \in V
$$

A proof of Korn's inequality may be found in [11, page 79]. On $V$ we consider the inner product and the associated norm given by

$$
(\mathbf{u}, \mathbf{v})_{V}=(\boldsymbol{\varepsilon}(\mathbf{u}), \boldsymbol{\varepsilon}(\mathbf{v}))_{Q}, \quad\|\mathbf{v}\|_{V}=\|\boldsymbol{\varepsilon}(\mathbf{v})\|_{Q} \quad \forall \mathbf{u}, \mathbf{v} \in V .
$$

It follows from Korn's inequality that $\|\cdot\|_{H_{1}}$ and $\|\cdot\|_{V}$ are equivalent norms on $V$ and therefore $\left(V,\|\cdot\|_{V}\right)$ is a real Hilbert space. Moreover, by the Sobolev trace theorem there 
exists a constant $c_{0}$, depending only on $\Omega, \Gamma_{1}$, and $\Gamma_{3}$, such that

$$
\|\mathbf{v}\|_{L^{2}\left(\Gamma_{3}\right)^{d}} \leq c_{0}\|\mathbf{v}\|_{V} \quad \forall \mathbf{v} \in V
$$

For the bonding field we will use the set

$$
\mathscr{Q}=\left\{\theta:[0, T] \longrightarrow L^{2}\left(\Gamma_{3}\right): 0 \leq \theta(t) \leq 1 \forall t \in[0, T] \text {, a.e. on } \Gamma_{3}\right\} .
$$

Finally, we consider the space of fourth-order tensor fields:

$$
\mathbf{Q}_{\infty}=\left\{\mathscr{E}=\left(\mathscr{E}_{i j k l}\right): \mathscr{E}_{i j k l}=\mathscr{E}_{j i k l}=\mathscr{E}_{k l i j} \in L^{\infty}(\Omega), 1 \leq i, j, k, l \leq d\right\},
$$

which is a real Banach space with the norm

$$
\|\mathscr{E}\|_{\mathbf{Q}_{\infty}}=\max _{0 \leq i, j, k, l \leq d}\left\|\mathscr{E}_{i j k l}\right\|_{L^{\infty}(\Omega)}
$$

We assume that the elasticity tensor $\mathscr{A}$ and the relaxation tensor $\mathscr{B}$ satisfy

$$
\mathscr{A} \in \mathbf{Q}_{\infty}
$$

$$
\begin{gathered}
\exists \alpha>0 \quad \text { such that } \mathscr{A} \boldsymbol{\xi} \cdot \boldsymbol{\xi} \geq \alpha\|\boldsymbol{\xi}\|^{2} \forall \boldsymbol{\xi} \in \mathbb{S}^{d} \text {, a.e. } \mathbf{x} \in \Omega \text {, } \\
\qquad \mathscr{B} \in C\left([0, T] ; \mathbf{Q}_{\infty}\right)
\end{gathered}
$$

The normal compliance function $p_{v}$ and the tangential function $p_{\tau}$ satisfy the assumptions

$$
\begin{aligned}
& p_{v}: \Gamma_{3} \times \mathbb{R} \longrightarrow \mathbb{R}_{+}, \\
& \exists L_{\nu}>0 \text { such that }\left|p_{\nu}\left(\mathbf{x}, r_{1}\right)-p_{\nu}\left(\mathbf{x}, r_{2}\right)\right| \leq L_{\nu}\left|r_{1}-r_{2}\right| \forall r_{1}, r_{2} \in \mathbb{R} \text {, a.e. } \mathbf{x} \in \Gamma_{3} \text {, } \\
& \left(p_{\nu}\left(\mathbf{x}, r_{1}\right)-p_{\nu}\left(\mathbf{x}, r_{2}\right)\right)\left(r_{1}-r_{2}\right) \geq 0 \quad \forall r_{1}, r_{2} \in \mathbb{R} \text {, a.e. } \mathbf{x} \in \Gamma_{3}, \\
& \text { the mapping } \mathbf{x} \longmapsto p_{\nu}(\mathbf{x}, r) \text { is measurable on } \Gamma_{3} \text {, for any } r \in \mathbb{R} \text {, } \\
& p_{\nu}(\mathbf{x}, r)=0 \quad \forall r \leq 0 \text {, a.e. } \mathbf{x} \in \Gamma_{3} \text {, } \\
& p_{\tau}: \Gamma_{3} \times \mathbb{R} \longrightarrow \mathbb{R}_{+}, \\
& \exists M_{\tau}>0 \text { such that }\left|p_{\tau}(\mathbf{x}, \beta)\right| \leq M_{\tau} \forall \beta \in \mathbb{R} \text {, a.e. } \mathbf{x} \in \Gamma_{3} \text {, } \\
& \text { the mapping } \mathbf{x} \longmapsto p_{\tau}(\mathbf{x}, 0) \text { belongs to } L^{2}\left(\Gamma_{3}\right) \text {. }
\end{aligned}
$$


8 Viscoelastic frictionless contact problems with adhesion

We also suppose that the body forces and surface tractions have the regularity

$$
\mathbf{f}_{0} \in C\left([0, T] ; L^{2}(\Omega)^{d}\right), \quad \mathbf{f}_{2} \in C\left([0, T] ; L^{2}\left(\Gamma_{2}\right)^{d}\right) .
$$

The adhesion coefficients satisfy

$$
\gamma_{\nu}, \gamma_{\tau} \in L^{\infty}\left(\Gamma_{3}\right), \quad \epsilon_{a} \in L^{2}\left(\Gamma_{3}\right), \quad \gamma_{\nu}, \gamma_{\tau}, \epsilon_{a} \geq 0 \text {, a.e. on } \Gamma_{3}
$$

and, finally, the initial bonding field satisfies

$$
\beta_{0} \in L^{2}\left(\Gamma_{3}\right), \quad 0 \leq \beta_{0} \leq 1 \text {, a.e. on } \Gamma_{3} .
$$

Next, we denote by $\mathbf{f}:[0, T] \rightarrow V$ the function defined by

$$
(\mathbf{f}(t), \mathbf{v})_{V}=\int_{\Omega} \mathbf{f}_{0}(t) \cdot \mathbf{v} d x+\int_{\Gamma_{2}} \mathbf{f}_{2}(t) \cdot \mathbf{v} d a \quad \forall \mathbf{v} \in V \text {, a.e. } t \in(0, T),
$$

and we note that conditions (3.31) imply

$$
\mathbf{f} \in C([0, T] ; V)
$$

For the Signorini problem we use the convex subset of admissible displacements given by

$$
U=\left\{\mathbf{v} \in V: v_{v} \leq 0 \text { on } \Gamma_{3}\right\}
$$

as well as the adhesion functional $j_{\text {ad }}: L^{\infty}\left(\Gamma_{3}\right) \times V \times V \rightarrow \mathbb{R}$ defined by

$$
j_{\mathrm{ad}}(\beta, \mathbf{u}, \mathbf{v})=\int_{\Gamma_{3}}\left(-\gamma_{\nu} \beta^{2} R_{\nu}\left(u_{\nu}\right) v_{\nu}+p_{\tau}(\beta) \mathbf{R}_{\tau}\left(\mathbf{u}_{\tau}\right) \cdot \mathbf{v}_{\tau}\right) d a
$$

For the problem with normal compliance, in addition to the functional (3.37), we need the normal compliance functional $j_{\mathrm{nc}}: V \times V \rightarrow \mathbb{R}$ given by

$$
j_{\mathrm{nc}}(\mathbf{u}, \mathbf{v})=\int_{\Gamma_{3}} p_{\nu}\left(u_{\nu}\right) v_{\nu} d a
$$

By a standard procedure based on Green's formula (2.7) we can derive the following variational formulation of the Signorini contact problem (3.1)-(3.8). 
Problem 3.3. Find a displacement field $\mathbf{u}:[0, T] \rightarrow V$ and a bonding field $\beta:[0, T] \rightarrow$ $L^{\infty}\left(\Gamma_{3}\right)$ such that

$$
\begin{gathered}
\mathbf{u}(t) \in U, \quad(\mathscr{A} \boldsymbol{\varepsilon}(\mathbf{u}(t)), \boldsymbol{\varepsilon}(\mathbf{v}-\mathbf{u}(t)))_{Q}+\left(\int_{0}^{t} \mathscr{R}(t-s) \boldsymbol{\varepsilon}(\mathbf{u}(s)) d s, \boldsymbol{\varepsilon}(\mathbf{v}-\mathbf{u}(t))\right)_{Q} \\
+j_{\mathrm{ad}}(\beta(t), \mathbf{u}(t), \mathbf{v}-\mathbf{u}(t)) \geq(\mathbf{f}(t), \mathbf{v}-\mathbf{u}(t))_{V} \quad \forall \mathbf{v} \in U, t \in[0, T], \\
\dot{\beta}(t)=-\left(\beta(t)\left(\gamma_{\nu} R_{\nu}\left(u_{\nu}(t)\right)^{2}+\gamma_{\tau}\left\|\mathbf{R}_{\tau}\left(\mathbf{u}_{\tau}(t)\right)\right\|^{2}\right)-\epsilon_{a}\right)_{+} \quad \text { on } \Gamma_{3} \text {, a.e. } t \in(0, T), \\
\beta(0)=\beta_{0} .
\end{gathered}
$$

The variational formulation of the problem with normal compliance (3.13)-(3.20) is as follows.

Problem 3.4. Find a displacement field $\mathbf{u}:[0, T] \rightarrow V$ and a bonding field $\beta:[0, T] \rightarrow$ $L^{\infty}\left(\Gamma_{3}\right)$ such that

$$
\begin{gathered}
(\mathscr{A} \boldsymbol{\varepsilon}(\mathbf{u}(t)), \boldsymbol{\varepsilon}(\mathbf{v}))_{Q}+\left(\int_{0}^{t} \mathscr{B}(t-s) \boldsymbol{\varepsilon}(\mathbf{u}(s)) d s, \boldsymbol{\varepsilon}(\mathbf{v})\right)_{Q}+j_{\mathrm{ad}}(\beta(t), \mathbf{u}(t), \mathbf{v}) \\
+j_{\mathrm{nc}}(\mathbf{u}(t), \mathbf{v})=(\mathbf{f}(t), \mathbf{v})_{V} \quad \forall \mathbf{v} \in V, t \in[0, T], \\
\dot{\beta}(t)=-\left(\beta(t)\left(\gamma_{\nu} R_{\nu}\left(u_{\nu}(t)\right)^{2}+\gamma_{\tau}\left\|\mathbf{R}_{\tau}\left(\mathbf{u}_{\tau}(t)\right)\right\|^{2}\right)-\epsilon_{a}\right)_{+} \quad \text { on } \Gamma_{3} \text {, a.e. } t \in(0, T), \\
\beta(0)=\beta_{0} .
\end{gathered}
$$

Note that the variational Problems 3.3 and 3.4 are formulated in terms of displacement and bonding fields, since the stress field was eliminated. However, if the solution $(\mathbf{u}, \beta)$ of these variational problems is known, then the corresponding stress field $\sigma$ can be easily obtained by using the linear viscoelastic constitutive law (3.1) or (3.13).

Remark 3.5. We also note that, unlike in Problems 3.1 and 3.2, in the variational Problems 3.3 and 3.4 we do not need to impose explicitly the restriction $0 \leq \beta \leq 1$. Indeed, (3.40) and (3.43) guarantee that $\beta(\mathbf{x}, t) \leq \beta_{0}(\mathbf{x})$ and, therefore, assumption (3.33) shows that $\beta(\mathbf{x}, t) \leq 1$ for $t \geq 0$, a.e. $\mathbf{x} \in \Gamma_{3}$. On the other hand, if $\beta\left(\mathbf{x}, t_{0}\right)=0$ at time $t_{0}$, then it follows from (3.40) and (3.43) that $\dot{\beta}(\mathbf{x}, t)=0$ for all $t \geq t_{0}$ and, therefore, $\beta(\mathbf{x}, t)=0$ for all $t \geq t_{0}$, a.e. $\mathbf{x} \in \Gamma_{3}$. We conclude that $0 \leq \beta(\mathbf{x}, t) \leq 1$ for all $t \in[0, T]$, a.e. $\mathbf{x} \in \Gamma_{3}$.

The well-posedness of Problems 3.3 and 3.4 will be provided in Sections 4 and 5, respectively. In the proofs we use a number of inequalities involving the functionals $j_{\text {ad }}$ and $j_{\mathrm{nc}}$ that we present in what follows. Below in this section $\beta, \beta_{1}, \beta_{2}$ denote elements of $L^{2}\left(\Gamma_{3}\right)$ such that $0 \leq \beta, \beta_{1}, \beta_{2} \leq 1$ a.e. on $\Gamma_{3}, \mathbf{u}_{1}, \mathbf{u}_{2}$, and $\mathbf{v}$ represent elements of $V$ and $c>0$ represent generic constants which may depend on $\Omega, \Gamma_{1}, \Gamma_{3}, p_{\nu}, p_{\tau}, \gamma_{\nu}, \gamma_{\tau}$, and $L$. 
Viscoelastic frictionless contact problems with adhesion

First, we notice that $j_{\mathrm{ad}}$ and $j_{\mathrm{nc}}$ are linear with respect to the last argument and therefore

$$
j_{\mathrm{ad}}(\beta, \mathbf{u},-\mathbf{v})=-j_{\mathrm{ad}}(\beta, \mathbf{u}, \mathbf{v}), \quad j_{\mathrm{nc}}(\mathbf{u},-\mathbf{v})=-j_{\mathrm{nc}}(\mathbf{u}, \mathbf{v}) .
$$

Next, using (3.37), the properties of the truncation operators $R_{v}$ and $\mathbf{R}_{\tau}$ as well as assumption (3.30) on the function $p_{\tau}$, after some calculus we find

$$
j_{\mathrm{ad}}\left(\beta_{1}, \mathbf{u}_{1}, \mathbf{u}_{2}-\mathbf{u}_{1}\right)+j_{\mathrm{ad}}\left(\beta_{2}, \mathbf{u}_{2}, \mathbf{u}_{1}-\mathbf{u}_{2}\right) \leq c \int_{\Gamma_{3}}\left|\beta_{1}-\beta_{2}\right||| \mathbf{u}_{1}-\mathbf{u}_{2}|| d a
$$

and, by (3.24), we obtain

$$
j_{\mathrm{ad}}\left(\beta_{1}, \mathbf{u}_{1}, \mathbf{u}_{2}-\mathbf{u}_{1}\right)+j_{\mathrm{ad}}\left(\beta_{2}, \mathbf{u}_{2}, \mathbf{u}_{1}-\mathbf{u}_{2}\right) \leq c\left\|\beta_{1}-\beta_{2}\right\|_{L^{2}\left(\Gamma_{3}\right)}\left\|\mathbf{u}_{1}-\mathbf{u}_{2}\right\|_{V} .
$$

Similar computations, based on the Lipschitz continuity of $R_{v}, \mathbf{R}_{\tau}$, and $p_{\tau}$, show that the following inequality also holds:

$$
\left|j_{\mathrm{ad}}\left(\beta, \mathbf{u}_{1}, \mathbf{v}\right)-j_{\mathrm{ad}}\left(\beta, \mathbf{u}_{2}, \mathbf{v}\right)\right| \leq c\left\|\mathbf{u}_{1}-\mathbf{u}_{2}\right\|_{V}\|\mathbf{v}\|_{V}
$$

We now take $\beta_{1}=\beta_{2}=\beta$ in (3.47) to deduce

$$
j_{\mathrm{ad}}\left(\beta, \mathbf{u}_{1}, \mathbf{u}_{2}-\mathbf{u}_{1}\right)+j_{\mathrm{ad}}\left(\beta, \mathbf{u}_{2}, \mathbf{u}_{1}-\mathbf{u}_{2}\right) \leq 0 .
$$

Also, we take $\mathbf{u}_{1}=\mathbf{v}$ and $\mathbf{u}_{2}=\mathbf{0}$ in (3.49), then we use the equalities $R_{\nu}(0)=0, \mathbf{R}_{\tau}(\mathbf{0})=\mathbf{0}$, and (3.45) to obtain

$$
j_{\text {ad }}(\beta, \mathbf{v}, \mathbf{v}) \geq 0 \text {. }
$$

Now, we use (3.38) and find

$$
\left|j_{\mathrm{nc}}\left(\mathbf{u}_{1}, \mathbf{v}\right)-j_{\mathrm{nc}}\left(\mathbf{u}_{2}, \mathbf{v}\right)\right| \leq \int_{\Gamma_{3}}\left|p_{\nu}\left(u_{1 \nu}\right)-p_{\nu}\left(u_{2 v}\right)\right|\left|v_{\nu}\right| d a
$$

and therefore (3.29b) and (3.24) imply

$$
\left|j_{\mathrm{nc}}\left(\mathbf{u}_{1}, \mathbf{v}\right)-j_{\mathrm{nc}}\left(\mathbf{u}_{2}, \mathbf{v}\right)\right| \leq c\left\|\mathbf{u}_{1}-\mathbf{u}_{2}\right\|_{V}\|\mathbf{v}\|_{V}
$$

We use again (3.38) and get

$$
j_{\mathrm{nc}}\left(\mathbf{u}_{1}, \mathbf{u}_{2}-\mathbf{u}_{1}\right)+j_{\mathrm{nc}}\left(\mathbf{u}_{2}, \mathbf{u}_{1}-\mathbf{u}_{2}\right)=\int_{\Gamma_{3}}\left(p_{\nu}\left(u_{1 \nu}\right)-p_{\nu}\left(u_{2 v}\right)\right)\left(u_{2 v}-u_{1 \nu}\right) d a
$$

and therefore (3.29c) implies

$$
j_{\mathrm{nc}}\left(\mathbf{u}_{1}, \mathbf{u}_{2}-\mathbf{u}_{1}\right)+j_{\mathrm{nc}}\left(\mathbf{u}_{2}, \mathbf{u}_{1}-\mathbf{u}_{2}\right) \leq 0 \text {. }
$$


Take $\mathbf{u}_{1}=\mathbf{v}$ and $\mathbf{u}_{2}=\mathbf{0}$ in the previous inequality and use (3.29e), (3.45) to obtain

$$
j_{\mathrm{nc}}(\mathbf{v}, \mathbf{v}) \geq 0 \text {. }
$$

The inequalities (3.47)-(3.55) combined with equalities (3.45) will be used in various places in the rest of the paper.

\section{Analysis of the Signorini contact problem}

The main result in this section is the following existence and uniqueness result.

Theorem 4.1. Assume that (3.28a)-(3.28b) and (3.30)-(3.33) hold. Then Problem 3.3 has a unique solution $(\mathbf{u}, \beta)$ which satisfies

$$
\begin{gathered}
\mathbf{u} \in C([0, T] ; V), \\
\beta \in W^{1, \infty}\left(0, T ; L^{2}\left(\Gamma_{3}\right)\right) \cap \mathcal{2} .
\end{gathered}
$$

A triple $(\mathbf{u}, \boldsymbol{\sigma}, \beta)$ which satisfies (3.1), (3.39)-(3.41) is called a weak solution of the Signorini contact problem (Problem 3.1). We conclude that under the stated assumptions, problem (3.1)-(3.8) has a unique weak solution. The regularity of the weak solution is given by (4.1), (4.2) and, in terms of stress,

$$
\boldsymbol{\sigma} \in C\left([0, T] ; Q_{1}\right) .
$$

Indeed, it follows from (3.39) that $\operatorname{Div} \boldsymbol{\sigma}(t)+\mathbf{f}_{0}(t)=\mathbf{0}$ for all $t \in[0, T]$ and, therefore, the regularity (4.1) of $\mathbf{u}$, combined with (3.28a), (3.28b), and the regularity of $\mathbf{f}_{2}$ in (3.31), implies (4.3).

We turn now to the proof of Theorem 4.1 which will be carried out in several steps. To this end, we assume in the following that (3.28a), (3.28b), and (3.30)-(3.33) hold; below, $c$ denotes a generic positive constant which may depend on $\Omega, \Gamma_{1}, \Gamma_{3}, \mathscr{A}, \mathscr{B}, \gamma_{\nu}, \gamma_{\tau}, p_{\tau}, L$, and $T$ but does not depend on $t$ nor on the rest of the input data, and whose value may change from place to place. Moreover, for the sake of simplicity, we suppress, in what follows, the explicit dependence of various functions on $\mathbf{x} \in \Omega \cup \Gamma_{3}$.

Let $\mathscr{Z}$ denote the closed subset of the space $C\left([0, T] ; L^{2}\left(\Gamma_{3}\right)\right)$ defined by

$$
\mathscr{L}=\left\{\beta \in C\left([0, T] ; L^{2}\left(\Gamma_{3}\right)\right) \cap \mathscr{Q}: \beta(0)=\beta_{0}\right\}
$$

and let $\beta \in \mathscr{L}$ be given. In the first step, we consider the following variational problem.

Problem 4.2. Find a displacement field $\mathbf{u}_{\beta}:[0, T] \rightarrow V$ such that

$$
\begin{aligned}
\mathbf{u}_{\beta}(t) \in U, \quad\left(\mathscr{A} \boldsymbol{\varepsilon}\left(\mathbf{u}_{\beta}(t)\right), \boldsymbol{\varepsilon}\left(\mathbf{v}-\mathbf{u}_{\beta}(t)\right)\right)_{Q}+\left(\int_{0}^{t} \mathscr{B}(t-s) \boldsymbol{\varepsilon}(\mathbf{u}(s)) d s, \boldsymbol{\varepsilon}(\mathbf{v}-\mathbf{u}(t))\right)_{Q} \\
\quad+j_{\mathrm{ad}}\left(\beta(t), \mathbf{u}_{\beta}(t), \mathbf{v}-\mathbf{u}_{\beta}(t)\right) \geq\left(\mathbf{f}(t), \mathbf{v}-\mathbf{u}_{\beta}(t)\right)_{V} \quad \forall \mathbf{v} \in U, t \in[0, T] .
\end{aligned}
$$

We have the following result. 
Lemma 4.3. There exists a unique solution to Problem 4.2 and it satisfies $\mathbf{u}_{\beta} \in C([0, T] ; V)$.

Proof. For a given $\boldsymbol{\eta} \in C([0, T] ; Q)$ and $t \in[0, T]$ we consider the operator $A_{\beta}(t): V \rightarrow V$ and the element $\mathbf{f}_{\eta}(t) \in V$ defined by

$$
\begin{gathered}
\left(A_{\beta}(t) \mathbf{u}, \mathbf{v}\right)_{V}=(\mathscr{A} \boldsymbol{\varepsilon}(\mathbf{u}), \boldsymbol{\varepsilon}(\mathbf{v}))_{Q}+j_{\mathrm{ad}}(\beta(t), \mathbf{u}, \mathbf{v}) \quad \forall \mathbf{u}, \mathbf{v} \in V, \\
\left(f_{\eta}(t), \mathbf{v}\right)_{V}=(f(t), \mathbf{u})_{V}-(\boldsymbol{\eta}(t), \boldsymbol{\varepsilon}(\mathbf{v}))_{Q} \quad \forall \mathbf{v} \in V .
\end{gathered}
$$

We use (3.28), (3.49), and (3.48) to see that $A_{\beta}(t)$ is a strongly monotone Lipschitz continuous operator; therefore, since $U$ is a nonempty closed convex subset of $V$, it follows from standard results on elliptic variational inequalities that there exists a unique element $\mathbf{u}_{\beta \eta}(t)$ such that

$$
\mathbf{u}_{\beta \eta}(t) \in U, \quad\left(A_{\beta}(t) \mathbf{u}_{\beta \eta}(t), \mathbf{v}-\mathbf{u}_{\beta \eta}(t)\right)_{V} \geq\left(\mathbf{f}_{\eta}(t), \mathbf{v}-\mathbf{u}_{\beta \eta}(t)\right)_{V} \quad \forall \mathbf{v} \in U
$$

and, using (4.6), we find that

$$
\begin{aligned}
\mathbf{u}_{\beta \eta}(t) \in U, \quad\left(\mathscr{A} \boldsymbol{\varepsilon}\left(\mathbf{u}_{\beta \eta}(t)\right), \boldsymbol{\varepsilon}\left(\mathbf{v}-\mathbf{u}_{\beta \eta}(t)\right)\right)_{Q}+\left(\boldsymbol{\eta}(t), \boldsymbol{\varepsilon}\left(\mathbf{v}-\mathbf{u}_{\beta \eta}(t)\right)\right)_{Q} \\
\quad+j_{\mathrm{ad}}\left(\beta(t), \mathbf{u}, \mathbf{v}-\mathbf{u}_{\beta \eta}(t)\right) \geq\left(\mathbf{f}(t), \mathbf{v}-\mathbf{u}_{\beta \eta}(t)\right)_{V} \quad \forall \mathbf{v} \in U .
\end{aligned}
$$

Moreover, (3.28), (3.47), and (4.8), combined with the regularities $\mathbf{f} \in C([0, T] ; V), \boldsymbol{\eta} \in$ $C([0, T] ; Q)$, and $\beta \in C\left([0, T] ; L^{2}\left(\Gamma_{3}\right)\right)$, imply that the mapping $t \mapsto \mathbf{u}_{\beta \eta}$ is continuous from $[0, T]$ with values in $V$. We conclude that there exists a unique function $\mathbf{u}_{\beta \eta} \in$ $C([0, T] ; V)$ which solves $(4.8)$ for all $t \in[0, T]$.

We now define the operator $\Lambda_{\beta}: C([0, T] ; Q) \rightarrow C([0, T] ; Q)$ by

$$
\Lambda_{\beta} \boldsymbol{\eta}(t)=\int_{0}^{t} \mathscr{B}(t-s) \boldsymbol{\varepsilon}\left(\mathbf{u}_{\beta \eta}(s)\right) d s \quad \forall \boldsymbol{\eta} \in C([0, T] ; Q), t \in[0, T] .
$$

Let $\boldsymbol{\eta}_{1}, \boldsymbol{\eta}_{2} \in C([0, T] ; Q)$. A standard computation based on (3.28b) and (4.8) shows that

$$
\left\|\Lambda_{\beta} \boldsymbol{\eta}_{1}(t)-\Lambda_{\beta} \boldsymbol{\eta}_{2}(t)\right\|_{Q} \leq c \int_{0}^{t}\left\|\boldsymbol{\eta}_{1}(s)-\boldsymbol{\eta}_{2}(s)\right\|_{Q} d s \quad \forall t \in[0, T]
$$

which implies that a power $\Lambda^{m}$ of $\Lambda$ is a contraction on the space $C([0, T] ; Q)$. Thus, there exists a unique element $\boldsymbol{\eta}_{\beta} \in C([0, T] ; Q)$ such that

$$
\Lambda_{\beta} \boldsymbol{\eta}_{\beta}(t)=\boldsymbol{\eta}_{\beta}(t) \quad \forall t \in[0, T]
$$

Denote $\mathbf{u}_{\beta}=\mathbf{u}_{\beta \eta_{\beta}}$. It follows from (4.8)-(4.11) that $\mathbf{u}_{\beta}$ is a solution of Problem 4.2, which concludes the existence part in the lemma. The uniqueness part follows from the uniqueness of the fixed point of the operator $\Lambda_{\beta}$ combined with the unique solvability of the variational inequality $(4.8)$ at any $t \in[0, T]$.

In the second step, we use the displacement field $\mathbf{u}_{\beta}$ obtained in Lemma 4.3, denote by $u_{\beta v}, \mathbf{u}_{\beta \tau}$ its normal and tangential components on $\Gamma_{3}$, and consider the following initialvalue problem. 
Problem 4.4. Find a bonding field $\theta_{\beta}:[0, T] \rightarrow L^{2}\left(\Gamma_{3}\right)$ such that

$$
\begin{gathered}
\dot{\theta}_{\beta}(t)=-\left(\theta_{\beta}(t)\left(\gamma_{\nu} R_{\nu}\left(u_{\beta \nu}(t)\right)^{2}+\gamma_{\tau}\left\|\mathbf{R}_{\tau}\left(\mathbf{u}_{\beta \tau}(t)\right)\right\|^{2}\right)-\epsilon_{a}\right)_{+} \text {a.e. } t \in(0, T), \\
\theta_{\beta}(0)=\beta_{0} .
\end{gathered}
$$

We obtain the following result.

Lemma 4.5. There exists a unique solution to Problem 4.4 and it satisfies $\theta_{\beta} \in W^{1, \infty}(0, T$, $\left.L^{2}\left(\Gamma_{3}\right)\right) \cap 2$.

Proof. Consider the mapping $F_{\beta}:[0, T] \times L^{2}\left(\Gamma_{3}\right) \rightarrow L^{2}\left(\Gamma_{3}\right)$ defined by

$$
F_{\beta}(t, \theta)=-\left(\gamma_{\nu} \theta\left(R_{\nu}\left(u_{\beta \nu}(t)\right)^{2}+\gamma_{\tau}\left\|\mathbf{R}_{\tau}\left(\mathbf{u}_{\beta \tau}(t)\right)\right\|^{2}\right)-\epsilon_{a}\right)_{+},
$$

for all $t \in[0, T]$ and $\theta \in L^{2}\left(\Gamma_{3}\right)$. It follows from the properties of the truncation operators $R_{v}$ and $\mathbf{R}_{\tau}$ that $F_{\beta}$ is Lipschitz continuous with respect to the second argument, uniformly in time. Moreover, for any $\theta \in L^{2}\left(\Gamma_{3}\right)$, the mapping $t \mapsto F_{\beta}(t, \theta)$ belongs to $L^{\infty}\left(0, T ; L^{2}\left(\Gamma_{3}\right)\right)$. Using now a version of Cauchy-Lipschitz theorem (see, e.g., [16, page $48])$, we obtain the existence of a unique function $\theta_{\beta} \in W^{1, \infty}\left(0, T, L^{2}\left(\Gamma_{3}\right)\right)$ which solves (4.12). Also, the arguments used in Remark 3.5 show that $0 \leq \theta_{\beta}(t) \leq 1$ for all $t \in[0, T]$, a.e. on $\Gamma_{3}$. Therefore, from the definition of the set 2 , we find that $\theta_{\beta} \in 2$, which concludes the proof of the lemma.

It follows from Lemma 4.5 that for all $\beta \in \mathscr{L}$ the solution $\theta_{\beta}$ of Problem 4.4 belongs to $\mathscr{L}$, see (4.4). Therefore, we may consider the operator $\Lambda: \mathscr{L} \rightarrow \mathscr{L}$ given by

$$
\Lambda \beta=\theta_{\beta}
$$

The third step consists of the following result.

Lemma 4.6. There exists a unique element $\beta^{*} \in \mathscr{L}$ such that $\Lambda \beta^{*}=\beta^{*}$.

Proof. We show that, for a positive integer $m$, the mapping $\Lambda^{m}$ is a contraction on $\mathscr{L}$. To this end, suppose that $\beta_{i}$ are two functions in $\mathscr{Z}$ and denote by $\mathbf{u}_{i}, \theta_{i}$ the functions obtained in Lemmas 4.3 and 4.5, respectively, for $\beta=\beta_{i}, i=1,2$.

Let $t \in[0, T]$. We use (4.5), the properties of the operators $\mathscr{A}$ and $\mathscr{B}$, and (3.47) to deduce that

$$
\left\|\mathbf{u}_{1}(t)-\mathbf{u}_{2}(t)\right\|_{V} \leq c\left(\left\|\beta_{1}(t)-\beta_{2}(t)\right\|_{L^{2}\left(\Gamma_{3}\right)}+\int_{0}^{t}\left\|\mathbf{u}_{1}(s)-\mathbf{u}_{2}(s)\right\|_{V} d s\right)
$$

and, using Gronwall's inequality, it yields

$$
\left\|\mathbf{u}_{1}(t)-\mathbf{u}_{2}(t)\right\|_{V} \leq c \int_{0}^{t}\left\|\beta_{1}(s)-\beta_{2}(s)\right\|_{L^{2}\left(\Gamma_{3}\right)} d s .
$$

On the other hand, it follows from (4.12) that

$$
\theta_{i}(t)=\beta_{0}-\int_{0}^{t}\left(\theta_{i}(s)\left(\gamma_{\nu} R_{\nu}\left(u_{i v}(s)\right)^{2}+\gamma_{\tau}\left\|\mathbf{R}_{\tau}\left(\mathbf{u}_{i \tau}(s)\right)\right\|^{2}\right)-\epsilon_{a}\right)_{+} d s
$$


14 Viscoelastic frictionless contact problems with adhesion

and then

$$
\begin{aligned}
& \left\|\theta_{1}(t)-\theta_{2}(t)\right\|_{L^{2}\left(\Gamma_{3}\right)} \\
& \leq c \int_{0}^{t}\left\|\theta_{1}(s) R_{\nu}\left(u_{1 \nu}(s)\right)^{2}-\theta_{2}(s) R_{\nu}\left(u_{2 \gamma}(s)\right)^{2}\right\|_{L^{2}\left(\Gamma_{3}\right)} d s \\
& \quad+c \int_{0}^{t}\left\|\theta_{1}(s)\right\| \mathbf{R}_{\tau}\left(\mathbf{u}_{1 \tau}(s)\right)\left\|^{2}-\theta_{2}(s)\right\| \mathbf{R}_{\tau}\left(\mathbf{u}_{2 \tau}(s)\right)\left\|^{2}\right\|_{L^{2}\left(\Gamma_{3}\right)} d s .
\end{aligned}
$$

Using the definitions of $R_{\nu}$ and $\mathbf{R}_{\tau}$ and writing $\theta_{1}=\theta_{1}-\theta_{2}+\theta_{2}$ we get

$$
\left\|\theta_{1}(t)-\theta_{2}(t)\right\|_{L^{2}\left(\Gamma_{3}\right)} \leq c \int_{0}^{t}\left\|\theta_{1}(s)-\theta_{2}(s)\right\|_{L^{2}\left(\Gamma_{3}\right)} d s+c \int_{0}^{t}\left\|\mathbf{u}_{1}(s)-\mathbf{u}_{2}(s)\right\|_{L^{2}\left(\Gamma_{3}\right)^{d}} d s .
$$

By Gronwall's inequality, it follows that

$$
\left\|\theta_{1}(t)-\theta_{2}(t)\right\|_{L^{2}\left(\Gamma_{3}\right)} \leq c \int_{0}^{t}\left\|\mathbf{u}_{1}(s)-\mathbf{u}_{2}(s)\right\|_{L^{2}\left(\Gamma_{3}\right)^{d}} d s
$$

and, using (3.24) we obtain

$$
\left\|\theta_{1}(t)-\theta_{2}(t)\right\|_{L^{2}\left(\Gamma_{3}\right)} \leq c \int_{0}^{t}\left\|\mathbf{u}_{1}(s)-\mathbf{u}_{2}(s)\right\|_{V} d s .
$$

We use (4.14) and the estimate (4.21) to find

$$
\left\|\Lambda \beta_{1}(t)-\Lambda \beta_{2}(t)\right\|_{L^{2}\left(\Gamma_{3}\right)} \leq c \int_{0}^{t}\left\|\mathbf{u}_{1}(s)-\mathbf{u}_{2}(s)\right\|_{V} d s .
$$

We now combine (4.16) and (4.22) to see that

$$
\left\|\Lambda \beta_{1}(t)-\Lambda \beta_{2}(t)\right\|_{L^{2}\left(\Gamma_{3}\right)} \leq c \int_{0}^{t}\left\|\beta_{1}(s)-\beta_{2}(s)\right\|_{L^{2}\left(\Gamma_{3}\right)} d s
$$

and, reiterating this inequality $m$ times, it yields

$$
\left\|\Lambda^{m} \beta_{1}-\Lambda^{m} \beta_{2}\right\|_{C\left([0, T] ; L^{2}\left(\Gamma_{3}\right)\right)} \leq \frac{c^{m} T^{m}}{m !}\left\|\beta_{1}-\beta_{2}\right\|_{C\left([0, T] ; L^{2}\left(\Gamma_{3}\right)\right)} .
$$

Recall that $\mathscr{L}$ is a nonempty closed set in the Banach space $C\left([0, T] ; L^{2}\left(\Gamma_{3}\right)\right)$ and note that inequality (4.24) shows that for $m$ sufficiently large $\Lambda^{m}: \mathscr{L} \rightarrow \mathscr{L}$ is a contraction. Then, by using the Banach fixed point theorem, it follows that $\Lambda$ has a unique fixed point $\beta^{*} \in \mathscr{L}$.

Now, we have all the ingredients to provide the proof of Theorem 4.1.

Proof. Existence. Let $\beta^{*} \in \mathscr{L}$ be the fixed point of $\Lambda$ and let $\mathbf{u}^{*}$ be the solution of Problem 4.2 for $\beta=\beta^{*}$, that is, $\mathbf{u}^{*}=\mathbf{u}_{\beta^{*}}$. Since $\theta_{\beta^{*}}=\beta^{*}$, we conclude by Lemmas 4.3 and 4.5 that $\left(\mathbf{u}^{*}, \beta^{*}\right)$ is a solution of Problem 3.3 and it satisfies (4.1), (4.2). 
Uniqueness. The uniqueness of the solution is a consequence of the uniqueness of the fixed point of operator $\Lambda$ defined by (4.14). Indeed, let $(\mathbf{u}, \beta)$ be a solution of Problem 3.3 which satisfies (4.1), (4.2). It follows from (3.39) that $\mathbf{u}$ is a solution to Problem 4.2 and, since by Lemma 4.3 this problem has a unique solution denoted by $\mathbf{u}_{\beta}$, we obtain

$$
\mathbf{u}=\mathbf{u}_{\beta}
$$

We replace $\mathbf{u}=\mathbf{u}_{\beta}$ in (3.40) and use the initial condition (3.41) to see that $\beta$ is a solution to Problem 4.4. Since by Lemma 4.5 this last problem has a unique solution denoted by $\theta_{\beta}$, we find

$$
\beta=\theta_{\beta}
$$

We use now (4.14) and (4.26) to see that $\Lambda \beta=\beta$, that is, $\beta$ is a fixed point of the operator $\Lambda$. It follows now from Lemma 4.6 that

$$
\beta=\beta^{*}
$$

The uniqueness part of the theorem is now a consequence of (4.25) and (4.27).

\section{Analysis of the problem with normal compliance}

The main result of this section is the following existence and uniqueness result.

Theorem 5.1. Assume that (3.28)-(3.33) hold. Then there exists a unique solution to Problem 3.4 with regularity (4.1), (4.2).

A triple $(\mathbf{u}, \boldsymbol{\sigma}, \beta)$ which satisfies (3.13), (3.42)-(3.44) is called a weak solution of the normal compliance contact problem (Problem 3.2). We conclude that, under the stated assumptions, problem (3.13)-(3.20) has a unique weak solution. Moreover, the weak solution satisfies (4.1)-(4.3), the regularity (4.3) being obtained by using arguments similar to those presented in Section 4.

Proof. The proof of Theorem 5.1 is similar to the proof of Theorem 4.1 and it is carried out in several steps. Since the modifications are straightforward, we omit the details. Below we use the set $\mathscr{L}$ defined in (4.4). The steps are the following.

(i) For each $\beta \in \mathscr{L}$ we prove that there exists a unique function $\mathbf{u}_{\beta} \in C([0, T] ; V)$ such that

$$
\begin{aligned}
& \left(\mathscr{A} \boldsymbol{\varepsilon}\left(\mathbf{u}_{\beta}(t)\right), \boldsymbol{\varepsilon}(\mathbf{v})\right)_{Q}+\left(\int_{0}^{t} \mathscr{B}(t-s) \boldsymbol{\varepsilon}\left(\mathbf{u}_{\beta}(s)\right) d s, \boldsymbol{\varepsilon}(\mathbf{v})\right)_{Q} \\
& \quad+j_{\mathrm{ad}}\left(\beta(t), \mathbf{u}_{\beta}(t), \mathbf{v}\right)+j_{\mathrm{nc}}\left(\mathbf{u}_{\beta}(t), \mathbf{v}\right)=(\mathbf{f}(t), \mathbf{v})_{V} \quad \forall \mathbf{v} \in U, t \in[0, T] .
\end{aligned}
$$

To prove this step we use arguments similar to those used in the proof of Lemma 4.3. The main difference arises from the fact that now (4.7) is replaced by the equality

$$
\mathbf{u}_{\beta \eta}(t) \in V, \quad\left(A_{\beta}(t) \mathbf{u}_{\beta \eta}(t), \mathbf{v}\right)_{V}=\left(\mathbf{f}_{\eta}(t)\right)_{V} \quad \forall \mathbf{v} \in V
$$


where, for $\beta \in \mathscr{L}$ and $t \in[0, T], A_{\beta}(t): V \rightarrow V$ is the operator defined by

$$
\left(A_{\beta}(t) \mathbf{u}, \mathbf{v}\right)_{V}=(\mathscr{A} \boldsymbol{\varepsilon}(\mathbf{u}), \boldsymbol{\varepsilon}(\mathbf{v}))_{\mathrm{Q}}+j_{\mathrm{ad}}(\beta(t), \mathbf{u}, \mathbf{v})+j_{\mathrm{nc}}(\mathbf{u}, \mathbf{v}) \quad \forall \mathbf{u}, \mathbf{v} \in V .
$$

We use (3.28) and the properties (3.45)-(3.55) of the functionals $j_{\text {ad }}$ and $j_{\text {nc }}$ to see that $A_{\beta}(t)$ is again a strongly monotone Lipschitz continuous operator.

(ii) For a given $\beta \in \mathscr{E}$ we prove that there exists a unique element $\theta$ such that

$$
\begin{gathered}
\theta_{\beta} \in W^{1, \infty}\left(0, T ; L^{2}\left(\Gamma_{3}\right)\right) \cap \mathscr{L}, \\
\dot{\theta}_{\beta}(t)=-\left(\gamma_{\nu} \theta_{\beta}(t)\left(R_{\nu}\left(u_{\beta \nu}(t)\right)^{2}+\gamma_{\tau}\left\|\mathbf{R}_{\tau}\left(\mathbf{u}_{\beta \tau}(t)\right)\right\|^{2}\right)-\epsilon_{a}\right)_{+} \quad \text { a.e. } t \in(0, T), \\
\theta_{\beta}(0)=\beta_{0} .
\end{gathered}
$$

The proof of this step is based on Lemma 4.5.

(iii) The operator $\Lambda: \mathscr{L} \rightarrow \mathscr{L}$ given by

$$
\Lambda \beta=\theta_{\beta}
$$

has unique element $\beta^{*} \in \mathscr{L}$. The proof is based on estimates similar to those presented in Lemma 4.6 and the Banach fixed point theorem.

(iv) Let $\beta^{*} \in \mathscr{L}$ be the fixed point of $\Lambda$ and denote $\mathbf{u}^{*}=\mathbf{u}_{\beta^{*}}$, where $\mathbf{u}_{\beta^{*}}$ is the function obtained in step (i) for $\beta=\beta^{*}$. Then, we use (5.1)-(5.5) to see that $\left(\mathbf{u}^{*}, \beta^{*}\right)$ is the unique solution of Problem 3.4 and it satisfies (4.1), (4.2).

\section{A convergence result}

We consider in this section the contact problem with normal compliance and adhesion when the contact condition (3.17) is replaced with

$$
-\sigma_{\nu}=\frac{1}{\mu} p_{\nu}\left(u_{\nu}\right)-\gamma_{\nu} \beta^{2} R_{\nu}\left(u_{\nu}\right) \quad \text { on } \Gamma_{3} \times(0, T)
$$

Here $\mu>0$ is a penalization parameter which may be interpreted as a deformability coefficient of the foundation, and then $1 / \mu$ is the surface stiffness coefficient. Indeed, when $\mu$ is smaller the reaction force of the foundation to penetration is larger and so the same force will result in a smaller penetration, which means that the foundation is less deformable. When $\mu$ is larger the reaction force of the foundation to penetration is smaller, and so the foundation is less stiff and more deformable.

Our aim is to study the behavior of the solution when $\mu \rightarrow 0$ and to prove that in the limit we recover the solution of the Signorini problem with adhesion.

We assume that (3.28)-(3.33) hold and we use the notation $U, \mathbf{f}, j_{\mathrm{ad}}$, and $j_{\mathrm{nc}}$ introduced in Section 3. Moreover, we reinforce assumption (3.29e) with the condition

$$
p_{\nu}(\mathbf{x}, r)=0 \quad \text { iff } r \leq 0
$$

a.e. $\mathbf{x} \in \Gamma_{3}$. It follows from the results in Section 4 that the Signorini contact problem (3.1)-(3.8) has a unique weak solution $(\mathbf{u}, \boldsymbol{\sigma}, \beta)$, such that (4.1)-(4.3) hold. Moreover, the 
weak solution satisfies

$$
\begin{gathered}
\mathbf{u}(t) \in U, \quad(\mathcal{A} \boldsymbol{\varepsilon}(\mathbf{u}(t)), \boldsymbol{\varepsilon}(\mathbf{v}-\mathbf{u}(t)))_{Q}+(\boldsymbol{\eta}(t), \boldsymbol{\varepsilon}(\mathbf{v}-\mathbf{u}(t)))_{Q} \\
+j_{\mathrm{ad}}(\beta(t), \mathbf{u}(t), \mathbf{v}-\mathbf{u}(t)) \geq(\mathbf{f}(t), \mathbf{v}-\mathbf{u}(t))_{V} \quad \forall \mathbf{v} \in U, t \in[0, T], \\
\dot{\beta}(t)=-\left(\beta(t)\left(\gamma_{\nu} R_{\nu}\left(u_{\nu}(t)\right)^{2}+\gamma_{\tau}\left\|\mathbf{R}_{\tau}\left(\mathbf{u}_{\tau}(t)\right)\right\|^{2}\right)-\epsilon_{a}\right)_{+} \quad \text { on } \Gamma_{3}, \text { a.e. } t \in(0, T), \\
\beta(0)=\beta_{0},
\end{gathered}
$$

where $\boldsymbol{\eta} \in C([0, T] ; Q)$ is the function given by

$$
\boldsymbol{\eta}(t)=\int_{0}^{t} \mathscr{B}(t-s) \boldsymbol{\varepsilon}(\mathbf{u}(s)) d s \quad \forall t \in[0, T] .
$$

It also follows from the discussion in Section 5 that, for each positive $\mu$, the contact problem (3.13)-(3.16), (3.18)-(3.20), (6.1) has a unique weak solution $\left(\mathbf{u}_{\mu}, \boldsymbol{\sigma}_{\mu}, \beta_{\mu}\right)$ with the regularity expressed in (4.1)-(4.3). Moreover, the solution satisfies

$$
\begin{gathered}
\left(\mathscr{A} \boldsymbol{\varepsilon}\left(\mathbf{u}_{\mu}(t)\right), \boldsymbol{\varepsilon}(\mathbf{v})\right)_{Q}+\left(\boldsymbol{\eta}_{\mu}(t), \boldsymbol{\varepsilon}(\mathbf{v})\right)_{\mathrm{Q}}+j_{\mathrm{ad}}\left(\beta_{\mu}(t), \mathbf{u}_{\mu}(t), \mathbf{v}\right) \\
+\frac{1}{\mu} j_{\mathrm{nc}}\left(\mathbf{u}_{\mu}(t), \mathbf{v}\right)=(\mathbf{f}(t), \mathbf{v})_{V} \quad \forall \mathbf{v} \in V, t \in[0, T], \\
\dot{\beta}_{\mu}(t)=-\left(\beta_{\mu}(t)\left(\gamma_{\nu} R_{\nu}\left(u_{\mu \nu}(t)\right)^{2}+\gamma_{\tau}\left\|\mathbf{R}_{\tau}\left(\mathbf{u}_{\mu \tau}(t)\right)\right\|^{2}\right)-\epsilon_{a}\right)_{+} \quad \text { on } \Gamma_{3}, \text { a.e. } t \in(0, T), \\
\beta_{\mu}(0)=\beta_{0},
\end{gathered}
$$

where $\boldsymbol{\eta}_{\mu} \in C([0, T] ; Q)$ is the function given by

$$
\boldsymbol{\eta}_{\mu}(t)=\int_{0}^{t} \mathscr{B}(t-s) \boldsymbol{\varepsilon}\left(\mathbf{u}_{\mu}(s)\right) d s \quad \forall t \in[0, T]
$$

and $u_{\mu \nu}, \mathbf{u}_{\mu \tau}$ denote the normal and tangential components of $\mathbf{u}_{\mu}$, respectively.

The behavior of the solution $\left(\mathbf{u}_{\mu}, \beta_{\mu}\right)$ as $\mu \rightarrow 0$ is given in the following theorem.

Theorem 6.1. Assume (3.28)-(3.33) and (6.2). Then the solution $\left(\mathbf{u}_{\mu}, \beta_{\mu}\right)$ of problem (6.7)(6.10) converges to the solution $(\mathbf{u}, \beta)$ of problem (6.3)-(6.5), that is,

$$
\left\|\mathbf{u}_{\mu}(t)-\mathbf{u}(t)\right\|_{V}+\left\|\beta_{\mu}(t)-\beta(t)\right\|_{L^{2}\left(\Gamma_{3}\right)} \longrightarrow 0
$$

as $\mu \rightarrow 0$, for all $t \in[0, T]$.

In addition to the mathematical interest in the result above, it is important from the mechanical point of view, since it shows that the weak solution of the adhesive contact problem with a rigid obstacle may be approached as closely as one wishes by the solution of the adhesive contact problem with a deformable foundation, with a sufficiently small deformability coefficient. 
The proof of theorem is carried out in several steps. In the rest of the section we suppose that assumptions of Theorem 6.1 hold and we denote by $c$ a positive generic constant which does not depend on time or on $\mu$, and whose value may change from place to place.

In the first step we consider the following auxiliary problem of finding a displacement field $\tilde{\boldsymbol{u}}_{\mu}:[0, T] \rightarrow V$ such that

$$
\begin{aligned}
& \left(\mathscr{A} \boldsymbol{\varepsilon}\left(\tilde{\boldsymbol{u}}_{\mu}(t)\right), \boldsymbol{\varepsilon}(\mathbf{v})\right)_{\mathrm{Q}}+(\boldsymbol{\eta}(t), \boldsymbol{\varepsilon}(\mathbf{v}))_{\mathrm{Q}}+j_{\mathrm{ad}}\left(\beta(t), \tilde{\boldsymbol{u}}_{\mu}(t), \mathbf{v}\right) \\
& \quad+\frac{1}{\mu} j_{\mathrm{nc}}\left(\tilde{\boldsymbol{u}}_{\mu}(t), \mathbf{v}\right)=(\mathbf{f}(t), \mathbf{v})_{V} \quad \forall \mathbf{v} \in V, t \in[0, T] .
\end{aligned}
$$

This problem is an intermediate problem between the two above, since here $\boldsymbol{\eta}=\boldsymbol{\eta}(t)$ and $\beta=\beta(t)$ are known, taken from the Signorini problem. Using arguments similar to those in the proof of Theorem 5.1 we deduce that for each positive $\mu$, problem (6.12) has a unique solution which satisfies $\tilde{\boldsymbol{u}}_{\mu} \in C([0, T] ; V)$.

We have the following convergence result.

LeMma 6.2. As $\mu \rightarrow 0$,

$$
\left\|\tilde{\boldsymbol{u}}_{\mu}(t)-\mathbf{u}(t)\right\|_{V} \longrightarrow 0
$$

for all $t \in[0, T]$.

Proof. Let $t \in[0, T]$. We choose $\mathbf{v}=\tilde{\boldsymbol{u}}_{\mu}(t)$ in (6.12) and find

$$
\begin{gathered}
\left(\mathscr{A} \boldsymbol{\varepsilon}\left(\tilde{\boldsymbol{u}}_{\mu}(t)\right), \boldsymbol{\varepsilon}\left(\tilde{\boldsymbol{u}}_{\mu}(t)\right)\right)_{\mathrm{Q}}+\left(\boldsymbol{\eta}(t), \boldsymbol{\varepsilon}\left(\tilde{\boldsymbol{u}}_{\mu}(t)\right)\right)_{\mathrm{Q}}+j_{\mathrm{ad}}\left(\beta(t), \tilde{\boldsymbol{u}}_{\mu}(t), \tilde{\boldsymbol{u}}_{\mu}(t)\right) \\
+\frac{1}{\mu} j_{\mathrm{nc}}\left(\tilde{\boldsymbol{u}}_{\mu}(t), \tilde{\boldsymbol{u}}_{\mu}(t)\right)=\left(\mathbf{f}(t), \tilde{\boldsymbol{u}}_{\mu}(t)\right)_{V} \cdot
\end{gathered}
$$

We use now (3.50) and (3.55) in (6.14) to obtain

$$
\left(\mathscr{A} \boldsymbol{\varepsilon}\left(\tilde{\boldsymbol{u}}_{\mu}(t)\right), \boldsymbol{\varepsilon}\left(\tilde{\boldsymbol{u}}_{\mu}(t)\right)\right)_{Q}+\left(\boldsymbol{\eta}(t), \boldsymbol{\varepsilon}\left(\tilde{\boldsymbol{u}}_{\mu}(t)\right)\right)_{Q} \leq\left(\mathbf{f}(t), \tilde{\boldsymbol{u}}_{\mu}(t)\right)_{V}
$$

and, keeping in mind (3.28), we deduce that

$$
\left\|\tilde{\boldsymbol{u}}_{\mu}(t)\right\|_{V} \leq c\left(\|\boldsymbol{\eta}(t)\|_{Q}+\|\mathbf{f}(t)\|_{V}\right)
$$

Then, there exists $\tilde{\boldsymbol{u}}(t) \in V$ and a subsequence of $\left\{\tilde{\boldsymbol{u}}_{\mu}(t)\right\}_{\mu}$, still denoted by $\left\{\tilde{\boldsymbol{u}}_{\mu}(t)\right\}_{\mu}$, such that

$$
\tilde{\boldsymbol{u}}_{\mu}(t) \rightarrow \tilde{\boldsymbol{u}}(t) \quad \text { weakly in } V \text {, as } \mu \longrightarrow 0 .
$$

Using (6.14) and (3.50) again we obtain

$$
j_{\mathrm{nc}}\left(\tilde{\boldsymbol{u}}_{\mu}(t), \tilde{\boldsymbol{u}}_{\mu}(t)\right) \leq c \mu .
$$

It follows now from (6.17) that

$$
\tilde{\boldsymbol{u}}_{\mu}(t) \longrightarrow \tilde{\boldsymbol{u}}(t) \quad \text { in } L^{2}\left(\Gamma_{3}\right)^{d} \text { as } \mu \longrightarrow 0
$$


and, recalling (3.38) for $j_{\mathrm{nc}}$ and using (6.18) and (6.19), we find that in the limit

$$
\int_{\Gamma_{3}} p_{\nu}\left(\tilde{u}_{\nu}(t)\right) \tilde{u}_{\nu}(t) d a=0
$$

Since the integrand is nonnegative, (3.29c), we obtain

$$
p_{\nu}\left(\tilde{u}_{\nu}(t)\right) \tilde{u}_{\nu}(t)=0 \quad \text { a.e. on } \Gamma_{3} \text {. }
$$

It follows now from $(6.2)$ that $\tilde{u}_{v}(t) \leq 0$ a.e. on $\Gamma_{3}$ which shows that $\tilde{\boldsymbol{u}}(t) \in U$.

By choosing the test functions $\mathbf{v}-\tilde{\boldsymbol{u}}_{\mu}(t)$ in (6.12) and keeping in mind the properties (3.29c) and (3.29e) of the normal compliance function $p_{\nu}$, we obtain

$$
\begin{aligned}
& \left(\mathscr{A} \boldsymbol{\varepsilon}\left(\tilde{\boldsymbol{u}}_{\mu}(t)\right), \boldsymbol{\varepsilon}\left(\mathbf{v}-\tilde{\boldsymbol{u}}_{\mu}(t)\right)\right)_{Q}+\left(\boldsymbol{\eta}(t), \boldsymbol{\varepsilon}\left(\mathbf{v}-\tilde{\boldsymbol{u}}_{\mu}(t)\right)\right)_{Q} \\
& \quad+j_{\mathrm{ad}}\left(\beta(t), \tilde{\boldsymbol{u}}_{\mu}(t), \mathbf{v}-\tilde{\boldsymbol{u}}_{\mu}(t)\right) \geq\left(\mathbf{f}(t), \mathbf{v}-\tilde{\boldsymbol{u}}_{\mu}(t)\right)_{V} \quad \forall \mathbf{v} \in U .
\end{aligned}
$$

Next, we use (3.37), (6.19), and the properties of $R_{v}, \mathbf{R}_{\tau}$, and $p_{\tau}$ to deduce that

$$
j_{\mathrm{ad}}\left(\beta(t), \tilde{\boldsymbol{u}}_{\mu}(t), \mathbf{v}-\tilde{\boldsymbol{u}}_{\mu}(t)\right) \longrightarrow j_{\mathrm{ad}}(\beta(t), \tilde{\boldsymbol{u}}(t), \mathbf{v}-\tilde{\boldsymbol{u}}(t)) \quad \text { as } \mu \longrightarrow 0,
$$

for any $\mathbf{v} \in V$. Therefore, by (6.22), (6.17), (6.23) and a lower-semicontinuity argument we find that

$$
\begin{aligned}
\tilde{\boldsymbol{u}}(t) \in U, \quad(\mathscr{A} \boldsymbol{\varepsilon}(\tilde{\boldsymbol{u}}(t)), \boldsymbol{\varepsilon}(\mathbf{v}-\tilde{\boldsymbol{u}}(t)))_{Q}+(\boldsymbol{\eta}(t), \boldsymbol{\varepsilon}(\mathbf{v})-\boldsymbol{\varepsilon}(\tilde{\boldsymbol{u}}(t)))_{Q} \\
\quad+j_{\mathrm{ad}}(\beta(t), \tilde{\boldsymbol{u}}(t), \mathbf{v}-\tilde{\boldsymbol{u}}(t)) \geq(\mathbf{f}(t), \mathbf{v}-\tilde{\boldsymbol{u}}(t))_{V} \quad \forall \mathbf{v} \in U .
\end{aligned}
$$

We choose now $\mathbf{v}=\mathbf{u}(t)$ in (6.24) and $\mathbf{v}=\tilde{\boldsymbol{u}}(t)$ in (6.3), add the two inequalities, and use the properties (3.28) of the elasticity operator $\mathscr{A}$ to obtain

$$
c\|\tilde{\boldsymbol{u}}(t)-\mathbf{u}(t)\|_{V}^{2} \leq j_{\mathrm{ad}}(\beta(t), \tilde{\boldsymbol{u}}(t), \mathbf{u}(t)-\tilde{\boldsymbol{u}}(t))+j_{\mathrm{ad}}(\beta(t), \mathbf{u}(t), \tilde{\boldsymbol{u}}(t)-\mathbf{u}(t)) .
$$

By (3.49) the right-hand side of this inequality is nonpositive and, thus,

$$
\tilde{\boldsymbol{u}}(t)=\mathbf{u}(t)
$$

We conclude that $\mathbf{u}(t)$ is the unique weak limit in $V$ of any subsequence of the sequence $\left\{\tilde{\boldsymbol{u}}_{\mu}(t)\right\}_{\mu}$ and, therefore, we find that the whole sequence of functions $\left\{\tilde{\boldsymbol{u}}_{\mu}(t)\right\}_{\mu}$ converges weakly to the element $\mathbf{u}(t) \in U$.

Using (6.22) with $\mathbf{v}=\mathbf{u}(t)$ and assumption (3.28) on $\mathscr{A}$ we deduce that

$$
\begin{aligned}
c\left\|\tilde{\boldsymbol{u}}_{\mu}(t)-\mathbf{u}(t)\right\|_{V}^{2} \leq & \left(\boldsymbol{\eta}(t), \boldsymbol{\varepsilon}\left(\mathbf{u}(t)-\tilde{\boldsymbol{u}}_{\mu}(t)\right)\right)_{Q}+\left(\mathbf{f}(t), \tilde{\boldsymbol{u}}_{\mu}(t)-\mathbf{u}(t)\right)_{V} \\
& +j_{\mathrm{ad}}\left(\beta(t), \tilde{\boldsymbol{u}}_{\mu}(t), \mathbf{u}(t)-\tilde{\boldsymbol{u}}_{\mu}(t)\right) \\
& +\left(\mathscr{A} \boldsymbol{\varepsilon}(\mathbf{u}(t)), \boldsymbol{\varepsilon}\left(\mathbf{u}(t)-\tilde{\boldsymbol{u}}_{\mu}(t)\right)\right)_{Q}
\end{aligned}
$$


Viscoelastic frictionless contact problems with adhesion

Taking into account (6.17), (6.23), and (6.26) we obtain from the previous inequality that

$$
\left\|\tilde{\boldsymbol{u}}_{\mu}(t)-\mathbf{u}(t)\right\|_{V} \longrightarrow 0 \quad \text { as } \mu \longrightarrow 0,
$$

which concludes the proof.

The next step in the proof of the theorem is the following.

Lemma 6.3. There exists a positive constant $c$, independent of $\mu$, such that

$$
\left\|\tilde{\boldsymbol{u}}_{\mu}(t)-\mathbf{u}_{\mu}(t)\right\|_{V} \leq c\left(\left\|\boldsymbol{\eta}_{\mu}(t)-\boldsymbol{\eta}(t)\right\|_{Q}+\left\|\beta_{\mu}(t)-\beta(t)\right\|_{L^{2}\left(\Gamma_{3}\right)}\right)
$$

for all $t \in[0, T]$.

Proof. Let $t \in[0, T]$. We find from (6.7) and (6.12) that

$$
\begin{gathered}
\left(\mathscr{A} \boldsymbol{\varepsilon}\left(\mathbf{u}_{\mu}(t)-\tilde{\boldsymbol{u}}_{\mu}(t)\right), \boldsymbol{\varepsilon}\left(\tilde{\boldsymbol{u}}_{\mu}(t)-\mathbf{u}_{\mu}(t)\right)\right)_{\mathrm{Q}}+\left(\boldsymbol{\eta}_{\mu}(t)-\boldsymbol{\eta}(t), \boldsymbol{\varepsilon}\left(\tilde{\boldsymbol{u}}_{\mu}(t)-\mathbf{u}_{\mu}(t)\right)\right)_{\mathrm{Q}} \\
+j_{\mathrm{ad}}\left(\beta_{\mu}(t), \mathbf{u}_{\mu}(t), \tilde{\boldsymbol{u}}_{\mu}(t)-\mathbf{u}_{\mu}(t)\right)-j_{\mathrm{ad}}\left(\beta(t), \tilde{\boldsymbol{u}}_{\mu}(t), \tilde{\boldsymbol{u}}_{\mu}(t)-\mathbf{u}_{\mu}(t)\right) \\
+\frac{1}{\mu} j_{\mathrm{nc}}\left(\mathbf{u}_{\mu}(t), \tilde{\boldsymbol{u}}_{\mu}(t)-\mathbf{u}_{\mu}(t)\right)-\frac{1}{\mu} j_{\mathrm{nc}}\left(\tilde{\boldsymbol{u}}_{\mu}(t), \tilde{\boldsymbol{u}}_{\mu}(t)-\mathbf{u}_{\mu}(t)\right)=0 .
\end{gathered}
$$

We use (3.45), (3.47), and (3.54) to deduce that

$$
\begin{gathered}
j_{\mathrm{ad}}\left(\beta_{\mu}(t), \mathbf{u}_{\mu}(t), \tilde{\boldsymbol{u}}_{\mu}(t)-\mathbf{u}_{\mu}(t)\right)-j_{\mathrm{ad}}\left(\beta(t), \tilde{\boldsymbol{u}}_{\mu}(t), \tilde{\boldsymbol{u}}_{\mu}(t)-\mathbf{u}_{\mu}(t)\right) \\
\quad \leq c\left\|\beta_{\mu}(t)-\beta(t)\right\|_{L^{2}\left(\Gamma_{3}\right)}\left\|\tilde{\boldsymbol{u}}_{\mu}(t)-\mathbf{u}_{\mu}(t)\right\|_{V}, \\
j_{\mathrm{nc}}\left(\mathbf{u}_{\mu}(t), \tilde{\boldsymbol{u}}_{\mu}(t)-\mathbf{u}_{\mu}(t)\right)-j_{\mathrm{nc}}\left(\tilde{\boldsymbol{u}}_{\mu}(t), \tilde{\boldsymbol{u}}_{\mu}(t)-\mathbf{u}_{\mu}(t)\right) \leq 0 .
\end{gathered}
$$

We use the last two inequalities in (6.30) and combine the resulting inequality with (3.28) to obtain (6.29).

We are now in a position to prove Theorem 6.1.

Proof of Theorem 6.1. Let $t \in[0, T]$. Using the triangle inequality and (6.29) we obtain

$$
\begin{aligned}
\| \mathbf{u}_{\mu}(t) & -\mathbf{u}(t)\left\|_{V}+\right\| \beta_{\mu}(t)-\beta(t) \|_{L^{2}\left(\Gamma_{3}\right)} \\
& \leq\left\|\tilde{\boldsymbol{u}}_{\mu}(t)-\mathbf{u}(t)\right\|_{V}+c\left(\left\|\boldsymbol{\eta}_{\mu}(t)-\boldsymbol{\eta}(t)\right\|_{Q}+\left\|\beta_{\mu}(t)-\beta(t)\right\|_{L^{2}\left(\Gamma_{3}\right)}\right) .
\end{aligned}
$$

The definitions (6.6) and (6.10) of $\boldsymbol{\eta}$ and $\boldsymbol{\eta}_{\mu}$ combined with the properties (3.29) of $\mathscr{B}$ yield

$$
\left\|\boldsymbol{\eta}_{\mu}(t)-\boldsymbol{\eta}(t)\right\|_{Q} \leq c \int_{0}^{t}\left\|\mathbf{u}_{\mu}(s)-\mathbf{u}(s)\right\|_{V} d s .
$$

Next, we use (6.4), (6.5), (6.8), (6.9), and arguments similar to those used in the proof of (4.21) to find that

$$
\left\|\beta_{\mu}(t)-\beta(t)\right\|_{L^{2}\left(\Gamma_{3}\right)} \leq c \int_{0}^{t}\left\|\mathbf{u}_{\mu}(s)-\mathbf{u}(s)\right\|_{V} d s .
$$


Inserting (6.33) and (6.34) in (6.32) and applying Gronwall's lemma to the resulting inequality we obtain

$$
\begin{aligned}
\| \mathbf{u}_{\mu}(t) & -\mathbf{u}(t)\left\|_{V}+\right\| \beta_{\mu}(t)-\beta(t) \|_{L^{2}\left(\Gamma_{3}\right)} \\
& \leq\left\|\tilde{\boldsymbol{u}}_{\mu}(t)-\mathbf{u}(t)\right\|_{V}+c \int_{0}^{t}\left\|\tilde{\boldsymbol{u}}_{\mu}(s)-\mathbf{u}(s)\right\|_{V} d s .
\end{aligned}
$$

On the other hand, we note that (6.16) implies that

$$
\left\|\tilde{\boldsymbol{u}}_{\mu}(t)-\mathbf{u}(t)\right\|_{V} \leq c\left(\|\mathbf{f}(t)\|_{V}+\|\mathbf{u}(t)\|_{V}+\|\boldsymbol{\eta}(t)\|_{Q}\right) .
$$

Since (6.13) and (6.36) hold for all $t \in[0, T]$, it follows from the Lebesgue convergence theorem that, as $\mu \rightarrow 0$,

$$
\int_{0}^{t}\left\|\tilde{\boldsymbol{u}}_{\mu}(s)-\mathbf{u}(s)\right\|_{V} d s \longrightarrow 0
$$

The convergence result (6.11) is now a consequence of (6.13), (6.35), and (6.37).

We end this section with the remark that Theorem 6.1 implies the following convergence result, in terms of stress:

$$
\left\|\sigma_{\mu}(t)-\sigma(t)\right\|_{Q_{1}} \longrightarrow 0
$$

as $\mu \rightarrow 0$, for all $t \in[0, T]$. Indeed, let $t \in[0, T]$ and $\mu>0$; equalities

$$
\boldsymbol{\sigma}_{\mu}(t)=\mathscr{A} \boldsymbol{\varepsilon}\left(\mathbf{u}_{\mu}(t)\right)+\int_{0}^{t} \mathscr{B}(t-s) \boldsymbol{\varepsilon}\left(\mathbf{u}_{\mu}(s)\right) d s, \quad \boldsymbol{\sigma}(t)=\mathscr{A} \boldsymbol{\varepsilon}(\mathbf{u}(t))+\int_{0}^{t} \mathscr{B}(t-s) \boldsymbol{\varepsilon}(\mathbf{u}(s)) d s
$$

combined with the properties of the operators $\mathscr{A}$ and $\mathscr{B}$ yield

$$
\left\|\boldsymbol{\sigma}_{\mu}(t)-\boldsymbol{\sigma}(t)\right\|_{Q} \leq c|| \mathbf{u}_{\mu}(t)-\mathbf{u}(t) \|_{V}
$$

Also, since (6.3) and (6.7) imply $\operatorname{Div} \boldsymbol{\sigma}_{\mu}(t)=\operatorname{Div} \boldsymbol{\sigma}(t)=-\mathbf{f}_{0}(t)$ in $\Omega$, we deduce that

$$
\left\|\boldsymbol{\sigma}_{\mu}(t)-\boldsymbol{\sigma}(t)\right\|_{Q_{1}}=\left\|\boldsymbol{\sigma}_{\mu}(t)-\boldsymbol{\sigma}(t)\right\|_{Q}
$$

The convergence (6.38) is now a consequence of (6.11), (6.40), and (6.41).

\section{References}

[1] K. T. Andrews, L. Chapman, J. R. Fernández, M. Fisackerly, M. Shillor, L. Vanerian, and T. Van Houten, A membrane in adhesive contact, SIAM Journal on Applied Mathematics 64 (2003), no. $1,152-169$.

[2] K. T. Andrews and M. Shillor, Dynamic adhesive contact of a membrane, Advances in Mathematical Sciences and Applications 13 (2003), no. 1, 343-356.

[3] O. Chau, J. R. Fernández, M. Shillor, and M. Sofonea, Variational and numerical analysis of a quasistatic viscoelastic contact problem with adhesion, Journal of Computational and Applied Mathematics 159 (2003), no. 2, 431-465. 
[4] O. Chau, M. Shillor, and M. Sofonea, Dynamic frictionless contact with adhesion, Journal of Applied Mathematics and Physics (ZAMP) 55 (2004), no. 1, 32-47.

[5] M. Cocu and R. Rocca, Existence results for unilateral quasistatic contact problems with friction and adhesion, Mathematical Modelling and Numerical Analysis 34 (2000), no. 5, 981-1001.

[6] J. R. Fernández, M. Shillor, and M. Sofonea, Analysis and numerical simulations of a dynamic contact problem with adhesion, Mathematical and Computer Modelling 37 (2003), no. 12-13, $1317-1333$.

[7] M. Frémond, Équilibre de structures qui adhèrent à leur support, Comptes Rendus des Séances de l’Académie des Sciences. Série II 295 (1982), no. 11, 913-916.

[8] _ Adhérence des solides, Journal de Mécanique Théorique et Appliquée 6 (1987), no. 3, $383-407$.

[9] _ Non-Smooth Thermomechanics, Springer, Berlin, 2002.

[10] W. Han, K. L. Kuttler, M. Shillor, and M. Sofonea, Elastic beam in adhesive contact, International Journal of Solids and Structures 39 (2002), no. 5, 1145-1164.

[11] J. Nečas and I. Hlavaček, Mathematical Theory of Elastic and Elasto-Plastic Bodies: An Introduction, Studies in Applied Mechanics, vol. 3, Elsevier Scientific, Amsterdam, 1981.

[12] M. Raous, L. Cangémi, and M. Cocu, A consistent model coupling adhesion, friction, and unilateral contact, Computer Methods in Applied Mechanics and Engineering 177 (1999), no. 3-4, 383399.

[13] J. Rojek and J. J. Telega, Contact problems with friction, adhesion and wear in orthopaedic biomechanics. I: general developments, Journal of Theoretical and Applied Mechanics 39 (2001), no. 3, 655-677.

[14] J. Rojek, J. J. Telega, and S. Stupkiewicz, Contact problems with friction, adhesion and wear in orthopaedic biomechanics. II: numerical implementation and application to implanted knee joints, Journal of Theoretical and Applied Mechanics 39 (2001), no. 3, 679-706.

[15] M. Shillor, M. Sofonea, and J. J. Telega, Models and Analysis of Quasistatic Contact. Variational Methods, Lect. Notes Phys., vol. 655, Springer, Berlin, 2004.

[16] M. Sofonea, W. Han, and M. Shillor, Analysis and Approximation of Contact Problems with Adhesion or Damage, Pure and Applied Mathematics (Boca Raton), vol. 276, Chapman \& Hall/CRC Press, Florida, 2006.

[17] C. Talon and A. Curnier, A model of adhesion added to contact with friction, Contact Mechanics (Praia da Consolação, 2001) (J. A. C. Martins and M. D. P. Monteiro Marques, eds.), Solid Mech. Appl., vol. 103, Kluwer Academic, Dordrecht, 2002, pp. 161-168.

Mohamed Selmani: Département de Mathématiques, Université Ferhat Abbas, Route de Scipion, 19000 Sétif, Algérie

E-mail address: s_elmanih@yahoo.fr

Mircea Sofonea: Laboratoire de Mathématiques et Physique pour les Systèmes, Université de Perpignan, 52 Avenue Paul Alduy, 66860 Perpignan, France

E-mail address: sofonea@univ-perp.fr 